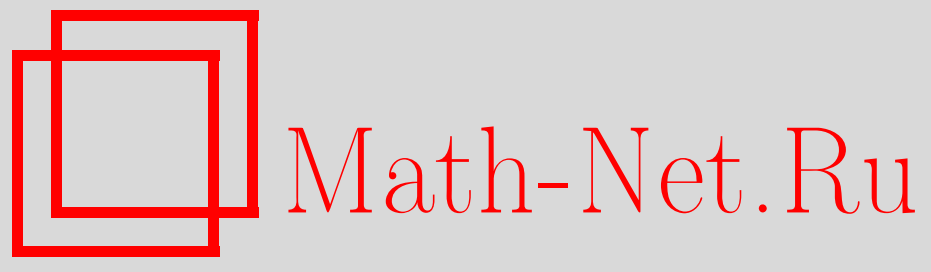

М. Шютт, Теоремы о сэндвичах для структур ШиодыИносы, Изв. РАН. Сер. матем., 2013, том 77, выпуск 1, 211-224

DOI: https://doi.org/10.4213/im7933

Использование Общероссийского математического портала Math-Net.Ru подразумевает, что вы прочитали и согласны с пользовательским соглашением http://www . mathnet.ru/rus/agreement

Параметры загрузки:

IP : 54.210 .77 .194

26 апреля 2023 г., 14:31:01

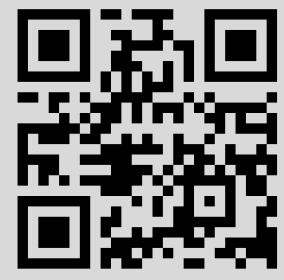




\section{М. Шютт}

\section{Теоремы о сэндвичах для структур Шиоды-Иносы}

Получена геометрическая реализация трех бесконечных семейств поверхностей типа K3, которые допускают сэндвич с поверхностями Куммера по отношению к структуре Шиоды-Иносы. Рассмотрен ряд конкретных примеров.

Библиография: 11 наименований.

Ключевые слова: поверхность типа К3, структура Шиоды-Иносы, эллиптическое расслоение, изогения.

DOI: $10.4213 / \operatorname{im} 7933$

\section{§1. Введение}

В последнее время в исследованиях по арифметике и геометрии поверхностей типа К3 важную роль играют структуры Шиоды-Иносы. Эти структуры позволяют установить естественную связь между специальными поверхностями типа К3 и поверхностями Куммера. В работе [1] Т. Шиода показал, что любая якобиева эллиптическая поверхность типа К3 с двумя особыми слоями типа II* допускает сэндвич с поверхностью Куммера (последняя имеет тип произведения). Впоследствии С. Ма в рамках теории Ходжа получил формальное доказательство того факта, что произвольная структура Шиоды-Иносы может быть достроена до сэндвича [2]. Однако для типичного случая, когда число Пикара поверхности равно 17, известны только пять явных геометрических примеров, построенных А. Кумаром [3], Б. ван Гименом, А. Сарти [4] и К. Койке [5].

В настоящей работе геометрически реализованы три бесконечные серии поверхностей типа К3 с числом Пикара не ниже 17, которые включаются в сэндвич по отношению к структуре Шиоды-Иносы.

Теорема 1. Пусть $N \in \mathbb{N}$. Предположим, что выполнена одна из трех следующих альтернатив:

1) $p \equiv 1(\bmod 4)$ для любого $p \mid N$;

2) $N=\prod_{i} p_{i}$ или $N=7 \prod_{i} p_{i}, p_{i} \equiv 1,2,4(\bmod 7)$ для любого $i$;

3) $N=\prod_{i} p_{i}$ или $N=15 \prod_{i} p_{i}$ для нечетного числа простых чисел $p_{i} \equiv$ $2,8(\bmod 15)$, либо $N=3 \prod_{i} p_{i}$ или $N=5 \prod_{i} p_{i}$ для четного числа простых чисел $p_{i} \equiv 2,8(\bmod 15)$.

Тогда для любой поверхности $X$ типа К3 с примитивным вложением $T_{X} \hookrightarrow U^{2}+\langle-2 N\rangle$ существует явная геометрически реализованная структура Шиодь-Иносъ, достраиваемая до сэндвича. 
Доказательство теоремы 1 состоит в рассмотрении трех различных семейств поверхностей типа K3 в $\S 3-5$ соответственно; завершения доказательств для каждой из альтернатив даны в пп. 3.5, 4.4, 5.4. Наша конструкция использует специализацию 4-мерных семейств поверхностей типа К3 посредством расширения решетки и эллиптических расслоений с сечением порядка 2. Следует отметить, что среди прочего эта конструкция позволяет реализовать все пять известных ранее примеров (следует рассмотреть дискриминанты; см. §2). Однако она не приводит к решеткам трансцендентных классов $U^{2}+\langle-2 N\rangle$, помимо тех, которые указаны в теореме 1 (см. п. 3.6).

Работа построена следующим образом. В 22 дан обзор необходимых фактов о структурах Шиоды-Иносы и сэндвичах. Каждый из последующих параграфов посвящен рассмотрению одного из исследуемых семейств. Всюду далее основным полем предполагается поле $\mathbb{C}$ комплексных чисел, хотя полученные уравнения имеют смысл над любым полем $k$, характеристика которого отлична от 2.

\section{§ 2. Структуры Шиоды-Иносы и конструкция сэндвича}

Классическим примером поверхности типа К3 является поверхность Куммера. Рассмотрим абелеву поверхность $A$ и профакторизуем ее по действию инволюции, определяемой групповой структурой. На факторпространстве имеется 16 рациональных простых двойных особенностей (типа $A_{1}$ ), разрешение которых приводит к поверхности типа $\mathrm{K} 3$, обозначаемой $\operatorname{Km}(A)$. Тем самым, имеется рациональное отображение степени 2

$$
A \rightarrow \operatorname{Km}(A)
$$

Эта реализация находит отражение в свойствах решеток трансцендентных классов, т. е. ортогональных дополнений $T_{X} \mathrm{k} \operatorname{NS}(X)$ в решетке $H^{2}(X, \mathbb{Z})$ относительно произведения классов когомологий. А именно, решетки трансцендентных классов подобны, т. е. их ранги совпадают, тогда как форма пересечения умножается на 2:

$$
T_{\mathrm{Km}(A)}=T_{A}(2) .
$$

$\mathrm{C}$ точки зрения классификации полезно связать с поверхностью $\operatorname{Km}(A)$ некоторую поверхность $X$ типа K3, решетка трансцендентных классов которой совпадает с исходной решеткой:

$$
T_{X}=T_{A}
$$

Впервые это удалось сделать Т. Шиоде и Х. Иносе в работе [6] для случая прямого произведения $A \cong E \times E^{\prime}$, где $E, E^{\prime}$ - эллиптические кривые. Предложенная ими конструкция применима к поверхностям типа К3 с числом Пикара 20 и существенно использует якобиевы эллиптические расслоения на поверхности $\operatorname{Km}\left(E \times E^{\prime}\right)$. Показано, что построенная поверхность $X$ допускает рациональное отображение на $\operatorname{Km}\left(E \times E^{\prime}\right)$ степени 2. Другими словами, $X$ обладает инволюцией Никулина (с восемью изолированными неподвижными точками), разрешение фактора по которой приводит к поверхности $\operatorname{Km}\left(E \times E^{\prime}\right)$. Следуя [7], в точности это условие обычно требуется в определении структуры 
Шиоды-Иносы произвольной комплексной абелевой поверхности $A$ и комплексной поверхности $X$ типа К3:

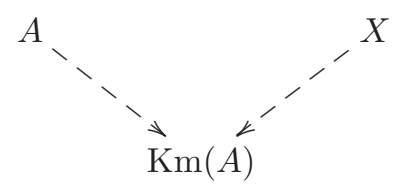

В случае неизогенных эллиптических кривых мы получаем $\mathrm{NS}(A)=U$ и $T_{A}=U^{2}$, где $U$ обозначает гиперболическую плоскость $\mathbb{Z}^{2}$ с формой пересечения $\left(\begin{array}{ll}0 & 1 \\ 1 & 0\end{array}\right)$. В типичном случае, в отличие от описанного, число Пикара поверхности $A$ равно единице. Если $A$ снабжена поляризацией степени $2 N$, $N \in \mathbb{N}$, имеет место равенство

$$
T_{A}=U^{2}+\langle-2 N\rangle
$$

В терминах решеток Д. Р. Моррисон получил исчерпывающий ответ на вопрос о том, когда поверхность типа К3 допускает структуру Шиоды-Иносы [7].

Теорема 2 (Д. Р. Моррисон). Алгебрачческая поверхность X muna К3 допускает структуру Шиодъ-Иносъ тогда и только тогда, когда для некоторого $N \in \mathbb{N}$ имеется примитивное вложение решеток

$$
T_{X} \hookrightarrow U^{2}+\langle-2 N\rangle .
$$

Эквивалентное условие состоит в том, что $X$ допускает инволюцию Никулина, переставляющую две ортогональные копии решетки $E_{8}$ в $\mathrm{NS}(X)$, или, более абстрактно, $E_{8}^{2} \hookrightarrow \mathrm{NS}(X)$. Напомним, что $E_{8}$ - это (единственная) унимодулярная четная отрицательно определенная решетка ранга 8.

Для обсуждения конструкции сэндвича нам потребуется вернуться к рассмотренному выше случаю прямого произведения $A=E \times E^{\prime}$ из статьи [6]. Т. Шиода заметил [1], что в этом случае сэндвич возникает автоматически. Последнее означает, что поверхность $\mathrm{Km}\left(E \times E^{\prime}\right)$ обладает инволюцией Никулина, которая, в свою очередь, определяет поверхность $X$. Тем самым, диаграмма (1) продолжается следующим образом в случае $A=E \times E^{\prime}$ :

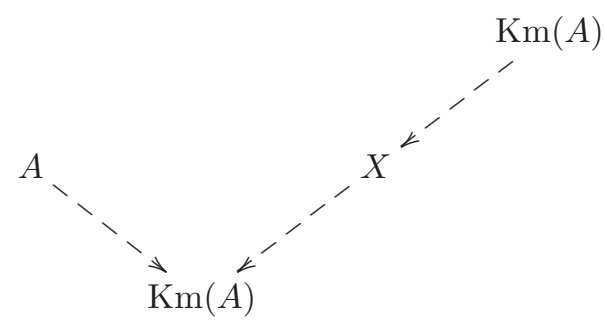

Для произвольной комплексной абелевой поверхности $A$ это наблюдение приводит к вопросу: всякая ли структура Шиоды-Иносы может быть продолжена до сэндвича? Положительный ответ был получен недавно в работе [2].

Теорема 3 (С. Ма). Любая структура Шиодъ-Иносъ допускает сэндвич. 
Отметим, что доказательство С. Ма основано на теории Ходжа. В частности, из него не удается извлечь никакой геометрической информации. В обозначениях теоремы 2 известны только пять значений $N \in \mathbb{N}$, для которых имеется явная геометрическая конструкция: случай $N=1$ рассматривается в [3] и отвечает поверхности Куммера якобианов кривых рода 2 ; случаи $N=2$ [4] и $N=3,5,7$ [5] отвечают эллиптическим поверхностям типа К3 с нулевым MW-рангом.

В каждом из случаев факторы реализованы посредством эллиптических расслоений с сечением порядка 2. Посредством общего слоя поверхности, $X$ и $\operatorname{Km}(A)$ можно интерпретировать как эллиптические кривые над полем $k(t)$. Тогда сечения переходят в $k(t)$-рациональные точки на общем слое; в частности, сечения порядка 2 соответствуют точкам порядка 2, что, тем самым, порождает изогении порядка 2 общих слоев, а также рациональные отображения порядка 2 между ассоциированными эллиптическими поверхностями. В настоящей работе мы используем преимущественно именно этот подход. Более точно, мы строим трехмерные семейства эллиптических расслоений MW-ранга 1 и сечения порядка 2, связанные со структурой Шиоды-Иносы. Теорема 1 утверждает, что эта конструкция позволяет получить бесконечный набор семейств поверхностей типа K3 со структурой сэндвича, и указанные выше пять семейств в этот набор входят. Для двух случаев с малым значением $N$, которые не вошли в указанный выше список примеров $(N=4,8)$, мы явно указываем уравнения, определяющие соответствующие семейства.

\section{§ 3. Первое семейство}

Рассмотрим 4-мерное семейство $\mathcal{X}$ поверхностей типа $\mathrm{K} 3$, для типичного члена $X$ которого выполнено

$$
\mathrm{NS}(X)=U+E_{7}^{2}
$$

Это семейство также недавно исследовалось в работе [8]. Оно может быть задано как эллиптическое расслоение с двумя особыми слоями кодаирова типа III* в точках 0 и $\infty$ :

$$
X: y^{2}=x^{3}+t^{3} a(t) x+t^{5} b(t)
$$

где $a(t), b(t) \in k[t]$ имеют степень 2 .

Здесь можно нормировать переменные $(x, y)$ и, независимо, переменную $t$, что позволяет избавиться от двух неопределенных коэффициентов. Гиперболическая плоскость $U \subset \mathrm{NS}(X)$ порождается нулевым сечением $O$ и типичным слоем $F$, в то время как решетки типа $E_{7}$ включают в себя компоненты слоев, не пересекающие $O$.

Естественно начать рассмотрение именно с этого семейства, поскольку оно специализируется в семейство Кумара с $\mathrm{NS}=U+E_{7}+E_{8}$, если положить, например, $\operatorname{deg}(a) \leqslant 1$. Решетку трансцендентных классов поверхности $X$ можно вычислить, используя теорию Никулина дискриминантных форм (или как 2-элементарную решетку):

$$
T_{X}=U^{2}+A_{1}^{2}
$$


Здесь мы рассматриваем двойственную решетку $L^{\vee}$ к невырожденной четной целочисленной решетке $L$. Она определяет дискриминантную группу $L^{\vee} / L$, которая является конечной абелевой группой порядка квадрат дискриминанта решетки $L$. Дискриминантная группа несет индуцированную с $L$ квадратичную форму, которая называется дискриминантной формой и обозначается через

$$
q_{L}: L^{\vee} / L \rightarrow \mathbb{Q} / 2 \mathbb{Z}
$$

Имеет место изоморфизм абелевых групп

$$
E_{7}^{\vee} / E_{7} \cong A_{1}^{\vee} / A_{1} \cong \mathbb{Z} / 2 \mathbb{Z}
$$

На самом деле, имеются порождающие элементы с квадратом $-3 / 2$ (соответственно, $-1 / 2)$, которые определяют непосредственное отождествление

$$
q_{E_{7}} \cong-q_{A_{1}} .
$$

3.1. Расширение решетки. Удобный способ специализации рассматриваемого 4-мерного семейства поверхностей типа K3 к подсемейству с числом Пикара $\rho \geqslant 17$ состоит в расширении решетки $L=\mathrm{NS}$ некоторым вектором $v$ из $T_{X}$ с отрицательным квадратом. В этом построении единственным тонким моментом является использование примитивного замыкания в К3-решетке $\Lambda$

$$
L^{\prime}:=\overline{\langle L, v\rangle} \subset \Lambda=H^{2}(X, \mathbb{Z})=U^{3}+E_{8}^{2} .
$$

Теория поляризованных поверхностей типа K3 гарантирует, что $L^{\prime}$ отвечает 3-мерному семейству (подсемейству), состоящему из поверхностей типа К 3 , для которых в общем положении имеем $\mathrm{NS}=L^{\prime}$ и $T^{\prime}=v^{\perp} \subset T_{X}$ для решетки трансцендентных классов. Поскольку мы интересуемся решетками трансцендентных классов, содержащими две копии $U$ (см. теорему 2), мы будем в дальнейшем расширять $L$ посредством вектора из $A_{1}^{2}$.

Пример 4. Рассмотрим вектор $v=(1,0) \in A_{1}^{2}$, т. е. порождающий первого экземпляра $A_{1}$. Изоморфизм (5) сопоставляет вектору $v / 2$ порождающий $w$ фактора $E_{7}^{\vee} / E_{7}$. Тем самым,

$$
L^{\prime}:=\overline{\langle L, v\rangle}=\langle L, v / 2+w\rangle
$$

На самом деле, мы присоединили по одной копии $E_{7}$ и $A_{1}$ к унимодуляной решетке $E_{8}$, так что $L^{\prime}=U+E_{7}+E_{8}$.

Поскольку расширение решетки включает в себя процедуру примитивного замыкания, мы должны выяснить, какие целые числа представляются решеткой $A_{1}^{2}$ примитивно, т. е. посредством вектора $v=\left(v_{1}, v_{2}\right)$ с условием НОД $\left(v_{1}, v_{2}\right)=1$. Следующая лемма является легким упражнением из теории квадратичных форм.

Лемма 5. Решетка $A_{1}^{2}$ представляет число $-2 N$ примитивно тогда и только тогда, когда $N$ является либо произведением простых чисел $p \equiv 1(\bmod 4)$, либо удвоенным произведением такого вида. 
Мы уже обсудили расширение решетки при $N=1$ (см. пример 4). Прочие случаи отвечают $v_{1} v_{2} \neq 0$, причем оба числа не являются одновременно четными. Пусть $w_{1}$ и $w_{2}$ - порождающие $E_{7}^{\vee} / E_{7}$ с квадратом $-3 / 2$, отвечающие соответствующим векторам из $A_{1}$ посредством (5). Тогда мы получаем, что

$$
L^{\prime}:=\overline{\left\langle L, v^{\prime}\right\rangle}, \quad v^{\prime}=\frac{v}{2}+\left\{\begin{array}{ll}
w_{1}, & v_{1} \text { нечетно, } \\
0, & v_{1} \text { четно }
\end{array}+ \begin{cases}w_{2}, & v_{2} \text { нечетно } \\
0, & v_{2} \text { четно }\end{cases}\right.
$$

Отметим, что в соответствии с нашими соглашениями $v^{\prime}$ является целым четным вектором:

$$
2 \mathbb{Z} \ni\left(v^{\prime}\right)^{2}=-\frac{N}{2}-\left\{\begin{array}{lll}
\frac{3}{2}, & \text { если } & N \text { нечетно, } \\
3, & \text { если } \quad N \text { четно. }
\end{array}\right.
$$

Напомним, что согласно общей теории эллиптических поверхностей с сечением группа NS порождается компонентами слоев и сечениями. Если $N=1$, то расширение решетки вызывает вырождение особых слоев, как в примере 4 . В случае $N \neq 1$ особые слои не вырождаются и дополнительный алгебраический класс $v^{\prime}$ можно отождествить с сечением $P$, которое ортогонально $O$ и $F$ в NS и пересекает один или оба слоя типа III* в нетождественной компоненте, в зависимости от четности $v_{1}$ и $v_{2}$. В терминах теории решеток Морделла-Вейля [9] вклад от $w_{1}, w_{2}$ в значение $\left(v^{\prime}\right)^{2}$ отвечает поправочным членам для соответствующих компонент слоев. Следовательно, $P$ имеет высоту $h(P)=N / 2$.

3.2. Решетка трансцендентных классов. По построению подсемейство с $L^{\prime} \hookrightarrow \mathrm{NS}$ имеет в общем положении решетку трансцендентных классов $T^{\prime}=$ $U^{2}+\langle-2 N\rangle$.

3.3. Альтернативное эллиптическое расслоение. Мы переходим к рассмотрению другого эллиптического расслоения на $X$, на котором имеется сечение порядка 2. Для этого мы отождествим дивизор $D$ кодаирова типа $I_{8}^{*}$ с носителем на $O$ и на компонентах слоев типа III*, как показано на рис. 1.

Линейная система дивизора $D$ индуцирует эллиптическое расслоение $X \rightarrow \mathbb{P}^{1}$. Здесь рациональные кривые, примыкающие к $D$, выступают в качестве нулевого сечения и сечения порядка два. Последнее утверждение легко проверяется посредством высотного спаривания, если заметить, что две оставшиеся компоненты исходных слоев типа III* (не пересекающиеся с дивизором типа I*) в общем положении находятся в двух слоях типа $\mathrm{I}_{2}$. Помимо этих трех особых слоев новое расслоение в общем положении имеет шесть слоев типа $\mathrm{I}_{1}$.

Явно дивизор $D$ может быть задан параметром $u=x / t^{2}$ в терминах (3). Элементарные преобразования приводят к нормальной форме Вейерштрасса

$$
X: y^{2}=t\left(u^{3} t^{2}+u a(t)+b(t)\right),
$$

т. е. эллиптической кривой над $k(u)$ с сечением $(0,0)$ порядка 2 . После расшире- 


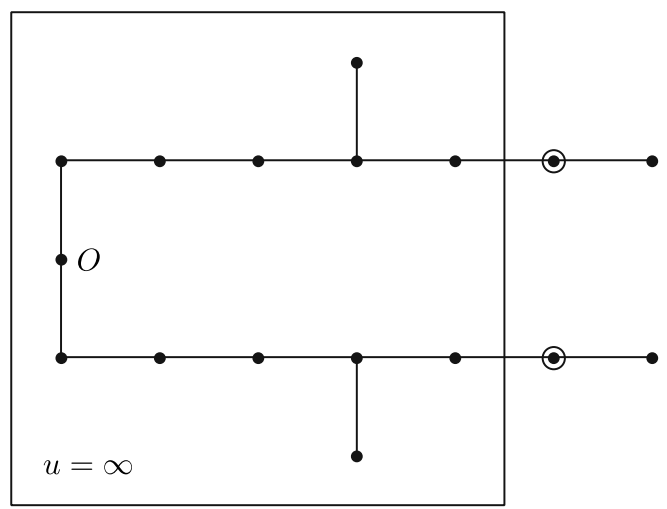

Рис. 1. Дивизор типа $\mathrm{I}_{8}^{*}$ с носителем на двух слоях типа III* и на сечении $O$

ния решетки рациональная кривая $P$ определяет мультисечение альтернативного расслоения, которое индуцирует сечение $P^{\prime}$ той же высоты $h\left(P^{\prime}\right)=N / 2$.

3.4. Поверхность Куммера. Рассмотрим 2-изогенную эллиптическую поверхность $Y$, возникающую из альтернативного эллиптического расслоения на $X$ посредством факторизации по сдвигу на $(0,0)$ и разрешения особенностей. В общем положении $Y$ имеет особые слои $\mathrm{I}_{4}^{*}, 2 \times \mathrm{I}_{1}, 6 \times \mathrm{I}_{2}$ и сечение порядка 2 . Решетку трансцендентных классов можно представить как $T_{Y}=(U(2))^{2}+A_{1}^{2}$. Рассмотрим специализацию $Y^{\prime}$ поверхности $Y$, отвечающую расширению решетки $X$ посредством сечения $P$ (и $P^{\prime}$ ).

ПредЛОЖЕНИЕ 6. Пусть $N$ - нечетное иелое число из леммы 5. Тогда $Y^{\prime}-$ поверхность Куммера, для которой $T_{Y^{\prime}}=T_{X}(2)=U(2)^{2}+\langle-4 N\rangle$.

ДокАзАтЕльство. Утверждение о том, что поверхность является поверхностью Куммера, следует из 2-делимости $T_{Y^{\prime}}$, что определяет четную решетку ранга не менее 17, как в теореме 2. Покажем, что решетка трансцендентных классов имеет указанный вид.

Сечение расширения $P^{\prime}$ поднимается до сечения $P^{*}$ на $Y$ высоты $h\left(P^{*}\right)=$ $2 h\left(P^{\prime}\right)=N$. Мы утверждаем, что это сечение не делится на 2 в $\mathrm{MW}\left(Y^{\prime}\right)$. Действительно, в противном случае существовало бы сечение $Q$, для которого $2 Q=P$, а значит, $h(Q)=N / 4$. Тогда, рассматривая имеющиеся особые слои, мы заключаем, что поправочные члены в высотном спаривании были бы все полуцелыми. Тем самым, $h(Q) \in \frac{1}{2} \mathbb{Z}$. Противоречие.

Итак, решетка $\mathrm{NS}\left(Y^{\prime}\right)$ порождена компонентами слоев, нулевым сечением и сечением порядка 2 вместе с $P^{*}$. В частности, используя [10, формула $\left.(22)\right]$, мы получаем

$$
\operatorname{disc} \operatorname{NS}\left(Y^{\prime}\right)=\frac{4 \times 2^{6}}{2^{2}} h\left(P^{*}\right)=2^{6} N .
$$

Далее мы используем тот факт, что прямой образ индуцирует вложение

$$
T_{X}(2) \hookrightarrow T_{Y^{\prime}}
$$


(которое в общем случае не обязано быть примитивным, но обе решетки имеют одинаковый ранг [11]; см. также [6], [7]). Согласно (7) обе решетки имеют одинаковый дискриминант, и тем самым они согласованы. Предложение доказано.

ЗАмечаниЕ 7 . Если число $N$ четно, то, напротив, сечение $P^{*}$ является 2-делимым в $\mathrm{MW}\left(Y^{\prime}\right)$ (достаточно рассмотреть 2-длину; см. также замечание 11). Тем самым, дискриминанты решеток $T_{X}(2)$ и $T_{Y^{\prime}}$ не совпадают. На самом деле, два случая расширения решеток в лемме 5 в определенном смысле двойственны: нечетное $N$ на $X$ отвечает четному $2 N$ на $Y$, и четное $N$ на $X$ определяет нечетное $N / 2$ на $Y$.

3.5. Доказательство теоремы 1,1$)$. Будем предполагать, что число $N$ разлагается в произведение простых чисел $p \equiv 1(\bmod 4)$, как в теореме 1,1$)$. Рассмотрим семейство поверхностей типа K3, заданное расширением решетки поверхности $\mathcal{X}$, отвечающим $N$. Согласно предложению 6 каждую поверхность $X^{\prime}$ из данного семейства с $\rho\left(X^{\prime}\right)=17$ можно включить в сэндвич по отношению к структуре Шиоды-Иносы, используя 2-изогению и ее двойственную. Остается исключить возможность того, что конструкция вырождается для большего числа Пикара.

Заметим, что каждый невырожденный член семейства обладает соответствующим эллиптическим расслоением с сечением порядка 2, но, возможно, с разными вырожденными слоями. Остается показать, что вне зависимости от числа Пикара фактор имеет 2-делимую решетку трансцендентных классов. Это вытекает из следующей леммы.

Лемма 8. Рассмотрим семейство поверхностей $(X, \iota)$ типа К3 с инволющией Никулина ᄂ. Предположим, что разрешение особенностей $Y$ фактора $X / \iota$ в общем положении имеет решетку трансцендентных классов

$$
T_{Y}=T_{X}(2) .
$$

Тогда это же имеет место для любой невырожденной специализации парь $(X, \iota)$.

ДокАзАТЕльство. Пусть $\left(X^{\prime}, \iota^{\prime}\right)$ - невырожденная специализация с фактором $Y^{\prime}$. Имеются естественные примитивные вложения

$$
T_{X^{\prime}} \hookrightarrow T_{X}, \quad T_{Y^{\prime}} \hookrightarrow T_{Y} .
$$

Используя прямой образ относительно морфизма факторизации и предположение леммы, мы получаем коммутативную диаграмму

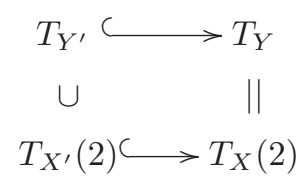

Рассматривая правый нижний угол, мы заключаем, что $T_{X^{\prime}}(2)$ примитивно вкладывается в $T_{Y}$. Рассматривая левый верхний угол, мы делаем аналогичный вывод для вложения $T_{X^{\prime}}(2) \subset T_{Y^{\prime}}$. Поскольку решетки имеют один и тот же ранг [11], мы получаем равенство $T_{Y^{\prime}}=T_{X^{\prime}}(2)$. Лемма доказана.

Таким образом, доказана теорема 1,1$)$. 
3.6. Оптимальность конструкции. Может возникнуть вопрос, нельзя ли получить посредством нашей конструкции другие поверхности типа К 3 , если не ограничивать себя рассмотрением прямого слагаемого $A_{1}^{2}$ в решетке $T$ из леммы 5. В самом деле, решетка трансцендентных классов $T$ представляет любое целое число, причем различными способами. Напомним, однако, что нашей целью является построение примитивных вложений

$$
U^{2}+\langle-2 N\rangle \hookrightarrow T
$$

Объясним, почему наша конструкция не приводит к подсемействам с решеткой трансцендентных классов, отличной от указанных в теореме 1. Для этого мы сначала вложим прямое слагаемое $U^{2}$ в $T$. В общем случае ортогональное дополнение $M=\left(U^{2}\right)^{\perp} \subset T$ не обязательно единственно, но род $M$, т. е. класс изогении, определяется по $T$ однозначно. Согласно теории групп классов род $M$ состоит из единственной решетки. Следовательно, для того чтобы изучать примитивные вложения (8), достаточно рассматривать представления $-2 N$ посредством $A_{1}^{2}$. Эти же аргументы можно использовать для двух нижеследующих конструкций (см. леммы 10, 13).

3.7. Другие расширения решеток. Для полноты изложения мы кратко обсудим другие расширения решеток семейства $\mathcal{X}$. Это также послужит дополнительной проверкой изложенных выше аргументов. Подобные рассуждения применимы и к семействам поверхностей типа K3, рассматриваемым в следующих параграфах.

Пусть $X^{\prime}$ - некоторая поверхность типа К3 с числом Пикара 17 и дискриминантом $2 N, N>1$, которая возникает из $\mathcal{X}$ посредством расширения решетки. Как и выше, $X^{\prime}$ обладает дополнительным сечением $P$ высоты $N / 2$, скажем, по отношению к исходному эллиптическому расслоению. Рассмотрим случай нечетного $N$. Здесь $P$ пересекает некоторые нетождественные компоненты слоев, тогда как для сечения $2 P$ высоты $2 N$ это не так. Обозначим через $\varphi$ ортогональную проекцию по отношению к $O, F$ в $\mathrm{NS}\left(X^{\prime}\right)$. По предположению $\varphi(2 P)$ ортогонально тривиальной решетке (т.е. образу $\mathrm{NS}(X)$ в $\mathrm{NS}\left(X^{\prime}\right)$ ), и мы получаем, что

$$
\varphi(2 P)^{2}=-2 N, \quad \varphi(2 P) \varphi(P)=-N .
$$

В частности, $\varphi(2 P) / N \in \mathrm{NS}\left(X^{\prime}\right)^{\vee}$. В дискриминантной группе $\mathrm{NS}\left(X^{\prime}\right)^{\vee} / \mathrm{NS}\left(X^{\prime}\right)$ это определяет элемент порядка $N$, где дискриминантная форма принимает значение $-2 / N$.

Предположим, что $T\left(X^{\prime}\right)=U^{2}+\langle-2 N\rangle-$ это одна из рассматриваемых решеток трансцендентных классов. Ее дискриминантная форма также принимает значение $-2 / N$ на элементе порядка $N$. Тогда для того, чтобы $\mathrm{NS}\left(X^{\prime}\right)$ и $T\left(X^{\prime}\right)$ были ортогональными дополнениями в К3-решетке, мы требуем, чтобы их дискриминатные формы имели противоположные знаки. В частности, это означает, что - 1 является квадратом по модулю любого простого делителя числа $N$. Тем самым, мы приходим к условиям теоремы 1.

В случае четного $N$ следует рассматривать два подслучая в зависимости от четности числа $M=N / 2$. Если $N / 2$ нечетно, то используем приведенные выше аргументы применительно к элементу $\varphi(P) / M \in \mathrm{NS}\left(X^{\prime}\right)^{\vee}$ порядка $N$ 
в дискриминантной группе. Если $N / 2$ четно, то $P$ лежит в узкой решетке Морделла-Вейля и пересекает все особые слои в тождественной компоненте. Тем самым, $\mathrm{NS}\left(X^{\prime}\right)=\mathrm{NS}(X)+\langle\varphi(P)\rangle$, и 2-длина дискриминантной группы равна 3 , что превосходит соответствующее значение для $U^{2}+\langle-2 N\rangle$. Противоречие.

\section{§4. Второе семейство}

В этом и следующем параграфах мы исследуем непосредственно эллиптические расслоения и сечение порядка 2, которое на этот раз является полустабильным. Такие расслоения допускают расширенную нормальную форму Вейершрасса

$$
y^{2}=x\left(x^{2}+a(t) x+b(t)\right)
$$

с приводимыми слоями в нулях многочлена $b(t)$. Мы рассматриваем два семейства, которые приводят к структурам Шиоды-Иносы. В настоящем параграфе будут изучаться поверхности с особыми слоями кодаировых типов $\mathrm{I}_{14}$ и $\mathrm{I}_{2}$. Соответствующие поверхности типа К3 образуют 4-мерное семейство поверхностей типа K3

$$
X: y^{2}=x\left(x^{2}+a(t) x+t\right),
$$

где $a \in k[t]$ имеет степень 4 и не обращается в нуль в точке $t=0$. Геометрически это означает, что $\rho(X)=16$.

4.1. Решетка трансцендентных классов. Решетка трансцендентных классов определяется альтернативным эллиптическим расслоением на поверхности $X$. А именно, легко определить дивизор кодаирова типа II* по $\mathrm{I}_{14}$ расширенной посредством $O$ и тождественной компоненты $\mathrm{I}_{2}$. Оставшиеся компоненты на $\mathrm{I}_{14}$ определяют сечение и $A_{6}$-конфигурацию рациональных кривых. Сравнивая ранги и дискриминанты, мы находим, что в общем положении

$$
\mathrm{NS}(X)=U+A_{6}+E_{8}, \quad T_{X}=U^{2}+\left(\begin{array}{ll}
-2 & -1 \\
-1 & -4
\end{array}\right) .
$$

Последнее представление легко проверяется по дискриминантной форме, поскольку $A_{6}^{\vee} / A_{6}$ обладает порождающим элементом с квадратом $-6 / 7$.

4.2. Факторсемейство. Рассмотрим фактор по действию сдвига на сечение порядка 2 и обозначим получившиеся эллиптические поверхности типа К3 через $Y$. Тогда в общем положении $Y$ имеет особые слои типов $\mathrm{I}_{7}, \mathrm{I}_{1}, 8 \times \mathrm{I}_{2}$, и $\mathrm{MW} \cong \mathbb{Z} / 2 \mathbb{Z}$.

Лемма 9. В общем положении $Y$ имеет трансцендентную решетку $T_{Y} \cong$ $T_{X}(2)$.

ДокАЗАтельство. Согласно [11] поверхность $Y$ типа К3 также имеет число Пикара 16, а значит, MW-ранг равен нулю по формуле Шиоды-Тейта. Стандартные формулы исключают другие кручения. Тем самым, группа $\mathrm{NS}(Y)$ порождена компонентами слоев и двумя сечениями конечных порядков. В частности, $\mathrm{NS}(Y)$ имеет дискриминант $2^{6} 7$. Тогда, как и выше, вложение $T_{X}(2) \hookrightarrow T_{Y}$, отвечающее прямому образу, является изометрией. 
4.3. Расширение решетки. Как и ранее, мы расширим решетку $\operatorname{NS}(X)$ посредством вектора из последнего слагаемого в $T_{X}$. В общем положении имеются следующие возможности.

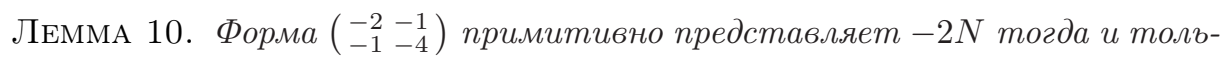
ко тогда, когда $N$ - произведение простых чисел $p \equiv 1,2,4(\bmod 7)$ или такое произведение, умноженное на 7.

Выберем вектор $v$, как в лемме 10, и расширим NS посредством порождающего элемента из $v^{\perp}$ в указанной выше решетке ранга 2. Мы приходим к решетке трансцендентных классов

$$
T^{\prime}=U^{2}+\mathbb{Z} v=U^{2}+\langle-2 N\rangle
$$

С точки зрения эллиптического расслоения это означает наличие дополнительного особого слоя $(N=1)$ или сечения $P$ высоты $2 N / 7(N>1)$.

4.4. Доказательство теоремы 1, 2). Пусть $N$ - число из теоремы 1,2 ) (или, эквивалентно, из леммы 10) и $X^{\prime}$ - член подсемейства поверхностей типа K3, соответствующего расширению решетки $X$, отвечающему числу $N$. По предположению решетка $T_{X^{\prime}}$ примитивно вкладывается в $U^{2}+\langle-2 N\rangle$. Более того, леммы 8, 10 показывают, что факторповерхность имеет решетку трансцендентных классов $T_{X^{\prime}}(2)$. Используя 2-изогению и двойственную к ней, мы заключаем, что эти поверхности образуют сэндвич по отношению к структуре Шиоды-Иносы.

ЗАмечание 11 . Из наших построений следует, что образ сечения $P$ на факторповерхности становится 2-делимым. Этот факт также можно проверить непосредственно на факторповерхности, сравнивая 2-длину группы NS и ранг решетки трансцендентных классов.

4.5. Пример $N=4$. Нам нужно снабдить эллиптическое расслоение (9) сечением высоты 8/7. С точностью до сдвига на сечение порядка 2 и инверсию такое сечение $P$ единственно; оно пересекает $\mathrm{I}_{14}$ в $\Theta_{4}$ (четвертая компонента) и $\mathrm{I}_{2}$ в тождественной компоненте, но не пересекает $O$. В самом деле, высотное спаривание дает

$$
h(P)=4-\frac{4 \cdot 10}{14}=\frac{8}{7}
$$

С учетом расширенной нормальной формы Вейерштрасса (9) сечение $P$ может иметь только вид $(\alpha, w)$, где $\alpha \in k^{*}$ и $w \in k[t]$ имеет степень 2 . Тогда пара $(\alpha, w)$ однозначно определяет многочлен $a(t)$ в $(9)$, поскольку

$$
w^{2}=\alpha^{3}+\alpha^{2} a(t)+\alpha t
$$

Мы получаем 3-мерное семейство поверхностей типа К3 с решеткой $T=$ $U^{2}+\langle-8\rangle$ в общем положении и унирациональным многообразием модулей. 


\section{§5. Третье семейство}

В качестве третьего семейства мы рассматриваем эллиптические поверхности типа К3 с сечением порядка 2 и особыми слоями типов $\mathrm{I}_{10}$ и $\mathrm{I}_{6}$. Здесь нормальная форма Вейерштрасса может быть преобразована к виду

$$
X: y^{2}=x\left(x^{2}+a(t) x+t^{3}\right)
$$

что вновь определяет 4-мерное семейство с $\rho=16$ в общем положении. Рассуждения в этом случае аналогичны рассуждениям в предыдущем случае, поэтому мы лишь наметим основные идеи.

5.1. Решетка трансцендентных классов. Дискриминантная форма позволяет найти решетку трансцендентных классов в общем положении

$$
T_{X}=U^{2}+\left(\begin{array}{ll}
-4 & -1 \\
-1 & -4
\end{array}\right)
$$

Вычисления можно упростить, используя сэндвичи с другим эллиптическим расслоением, например со слоями типов III* $^{*}$ и $\mathrm{IV}^{*}$ и сечением высоты $5 / 2$ (эллиптический параметр $u=x / t$ ). Это дает представление для $q_{\mathrm{NS}(X)}$ вида $\mathbb{Z} / 3 \mathbb{Z}(-4 / 3)+\mathbb{Z} / 5 \mathbb{Z}(-2 / 5)$, что согласуется с формой $q_{T_{X}}$ с точностью до знака.

5.2. Факторсемейство. Обозначим факторы эллиптических поверхностей с сечением порядка 2 через $Y$. Тогда в общем положении $Y$ имеет особые слои $\mathrm{I}_{5}, \mathrm{I}_{3}, 8 \times \mathrm{I}_{2}$, и $\mathrm{MW} \cong \mathbb{Z} / 2 \mathbb{Z}$.

ЛЕмма 12. В общем положснии $Y$ имеет решетку трансцендентных клас$\cos T_{Y} \cong T_{X}(2)$.

ДокАЗАтельство. Рассматривая особые слои и сечение конечного порядка, мы заключаем, что $\mathrm{NS}(Y)$ имеет дискриминант $2^{6} 15$. Как и выше, вложение $T_{X}(2) \hookrightarrow T_{Y}$ прямого образа является изометрией.

5.3. Расширение решетки. Расширим решетку $\mathrm{NS}(X)$ вектором из последнего слагаемого в $T_{X}$. Анализ того, какие числа могут быть примитивно представлены, в этом случае является несколько более деликатным. Это можно заметить уже из того факта, что числа 2, 3 и 5 представимы нужным образом, а числа 6,10 или 15 не представимы. Это связано с тем, что соответствующая квадратичная форма определяет класс порядка 2 в группе классов $\mathrm{Cl}(-15)$. Последнее объясняет появление условия четности.

Лемма 13. Целое число $-2 N$ представляется решеткой $\left(\begin{array}{l}-4 \\ -1 \\ -4\end{array}\right)$ примитивно тогда и только тогда, когда:

1) $N$ является произведением нечетного числа простых чисел $p \equiv$ 2,8 $(\bmod 15)$ или 15, умноженному на такое произведение;

2) $N$ равно 3 или 5, умноженному на произведение четного числа простых чисел $p \equiv 2,8(\bmod 15)$. 
Расширение NS посредством примитивного вектора, перпендикулярного вектору из леммы 13, приводит к решетке трансцендентных классов

$$
T^{\prime}=U^{2}+\langle-2 N\rangle
$$

При этом эллиптическое расслоение снабжено сечением $P$ высоты $2 N / 15$.

5.4. Доказательство теоремы $\mathbf{1}, \mathbf{3})$. Пусть $N$ - число из теоремы 1,3 ) (или, эквивалентно, из леммы 13) и $X^{\prime}$ - член подсемейства поверхностей типа K3, определенного расширением решетки $X$, отвечающим $N$. По предположению $T_{X^{\prime}}$ примитивно вкладывается в $U^{2}+\langle-2 N\rangle$. Более того, из лемм 8, 13 следует, что факторповерхность имеет решетку трансцендентных классов $T_{X^{\prime}}(2)$. Тем самым, эти поверхности реализуют сэндвич относительно структуры Шиоды-Иносы.

5.5. Пример $N=8$. Эллиптическое расслоение (10) должно иметь сечение $P$ высоты 16/15. Достаточно, чтобы $P$ пересекало $\mathrm{I}_{10}$ и $\mathrm{I}_{6}$ в $\Theta_{2}$ (вторая компонента) и не пересекало $O$, поскольку

$$
h(P)=4-\frac{2 \cdot 8}{10}-\frac{2 \cdot 4}{6}=\frac{16}{15} .
$$

Нормальная форма Вейерштрасса (10) показывает, что $P$ должно иметь вид $\left(\alpha t^{2}, w t^{2}\right)$, где $\alpha \in k^{*}$ и $w \in k[t]$ имеет степень 2. Тогда пара $(\alpha, w)$ однозначно определяет многочлен $a(t)$ в $(10)$, поскольку

$$
w^{2}=\alpha^{3} t^{2}+\alpha^{2} a(t)+\alpha t .
$$

Мы получаем 3-мерное семейство поверхностей типа $\mathrm{K} 3$ с решеткой $T=U^{2}+$ $\langle-16\rangle$ в общем положении и унирациональным многообразием модулей.

Идея написания этой статьи возникла у автора во время участия в семинаре по арифметике и геометрии поверхностей типа К3 и трехмерных многообразий Калаби-Яу в институте Филдса в августе 2011 г. Автор благодарит организаторов семинара за стимулирующую атмосферу и гостеприимство, кроме того, рецензента, а также переводчика И. В. Аржанцева за ценные замечания.

\section{Список литературы}

1. T. Shioda, "Kummer sandwich theorem of certain elliptic K3 surfaces", Proc. Japan Acad. Ser. A Math. Sci., 82:8 (2006), 137-140.

2. S. Ma, "On K3 surfaces which dominate Kummer surfaces", Proc. Amer. Math. Soc., 141:1 (2013), 131-137.

3. A. Kumar, "K3 surfaces associated with curves of genus two", Int. Math. Res. Not. IMRN, 2008, №6, Art. ID rnm165.

4. B. van Geemen, A. Sarti, "Nikulin involutions on K3 surfaces", Math. Z., 255:4 (2007), 731-753.

5. K. Koike, Elliptic K3 surfaces admitting a Shioda-Inose structure, arXiv: 1104.1470.

6. T. Shioda, H. Inose, "On singular K3 surfaces", Complex analysis and algebraic geometry, Iwanami Shoten, Tokyo, 1977, 119-136. 
7. D. R. Morrison, "On K3 surfaces with large Picard number", Invent. Math., 75:1 (1984), 105-121.

8. A. Clingher, C.F. Doran, "Note on a geometric isogeny of $K 3$ surfaces", Int. Math. Res. Not. IMRN, 2011, № 16, 3657-3687.

9. T. Shioda, "On the Mordell-Weil lattices", Comment. Math. Univ. St. Paul., 39:2 (1990), 211-240.

10. M. Schütt, T. Shioda, "Elliptic surfaces", Algebraic geometry in East Asia (Seoul, Korea, 2008), Adv. Stud. Pure Math., 60, Math. Soc. Japan, Tokyo, 2010, 51-160.

11. H. Inose, "Defining equations of singular $K 3$ surfaces and a notion of isogeny", Proceedings of the International Symposium on Algebraic Geometry (Kyoto, 1977), Kinokuniya Book Store, Tokyo, 1978, 495-502.

M. Шютт (M. Sснӥтт)

Leibniz University of Hannover, Germany

E-mail: schuett@math.uni-hannover.de

Перевод с английского И.В. Аржанщева
Поступило в редакцию

07.11 .2011 